\title{
Corneal wavefront guided versus aberration free transepithelial photorefractive keratectomy in myopic patients with high pre-existing corneal higher order aberrations
}

Amin Faisal Ellakwa ( $\square$ ellakwa@yahoo.com)

Menoufia University https://orcid.org/0000-0002-6661-2224

Marwa Ali Zaki

Menoufia University

Rana Mohammed Ashour

Menoufia University

Research article

Keywords:

Posted Date: May 7th, 2019

DOI: https://doi.org/10.21203/rs.2.458/v2

License: (c) (i) This work is licensed under a Creative Commons Attribution 4.0 International License.

Read Full License 


\section{Abstract}

Objectives to compare the efficacy, safety and predictability of corneal wavefront guided (WFG) and aberration free ablation in single-step transepithelial photorefractive keratectomy (TransPRK) in myopic patients with high pre-existing corneal higher order aberrations (HOAs). Background Corneal WFG and aberration free treatments have been proposed as methods to achieve better visual, refractive, and optical outcomes. Methods Non-randomized controlled trailwas conducted in El-HekmaEye-LASIK center, Menoufia governorate, Egypt during the period from January 2017 to December 2017. TransPRK was performed to eligible myopic patients with or without astigmatism with corneal HOAs $\geq 0.35 \mu$ utilizing either aberration free or corneal WFG patterns provided by ORKCAM software (SCHWIND eye-techsolutions, Kleinostheim, Germany). Uncorrected distance visual acuity (UDVA), manifest and cycloplegic refractions, best spectacle corrected distance visual acuity (CDVA), thorough slit lamp examination and corneal topography were assessed and repeated six months postoperatively. Results Six months postoperatively, $97.6 \%$ and $100 \%$ of patients achieved MRSE within $\pm 1 \mathrm{D}$ with $76.2 \%$ and $66.67 \%$ of patients achieved UDVA of $\geq 20 / 20$ in aberration free and corneal WFG groups respectively. Both groups showed increase in spherical and total corneal HOAs, with no significant difference in corneal WFG $(p=0.08, p=0.28$ respectively) while significantly higher postoperatively in aberration free group $(p<0.001$, $p=0.001$ respectively). Coma and trefoil aberrations declined after corneal WFG technique, yet increased after aberration free one. Conclusions both aberration free and corneal WFG TransPRK were safe, effective and predictable in treatment of myopia in patients with high pre-existing corneal HOAs with better aberrometric outcomes in corneal WFG group.

\section{Background}

Single-step transepithelial photorefractive keratectomy (TransPRK) has been proven as a good method to treat compound myopic astigmatism achieving comparable results to other surgical techniques, minimizing known drawbacks of photorefractive keratectomy (PRK) as postoperative pain, corneal haze, irregular epithelial healing and long recovery time and avoiding flap related complications ${ }^{(1)(2)}$.

Conventional ablation profiles previously used in PRK are believed to increase the optical aberrations postoperatively significantly(3)(4). Impaired visual performance (impaired low-contrast visual acuity and glare visual acuity) after PRK was correlated with the increase in the ocular aberrations ${ }^{(5)(6)}$.

This has urged the need to develop new ablation techniques to improve the optical outcome and then subsequently the visual outcome. Wavefront optimized (aberrationfree) is a non-individualized pattern aiming at prevention of induction new higher order aberrations (HOAs) mainly spherical aberrations ${ }^{(7)}$, was first proposed, followed by the $Q$ value customized aspherical treatment, that targets desired $Q$ value in order to preserve the corneal asphericity ${ }^{(8)}$. Ocular wavefront guided (ocular WFG) technique aims not only at minimizing the induction of new HOAs, but also correction of the pre-existing $\mathrm{HOAs}^{(9)}$. Another method was topography guided technique, that aims at reshaping the cornea into ideal shape based on data obtained from topography, taking into consideration the refractive error ${ }^{(10)}$. Finally, corneal WFG 
method was proposed which converts information from the topographer into a corneal wavefront map using Zernike polynomials expressing them as root main square values ${ }^{(11)(12)}$.

The cornea is responsible for $80 \%$ of the refractive power of the eye and the aberrometers can only measure aberrations at the pupil size. Also, internal ocular aberrations change with age, accommodation, and pupil size. Nonetheless, there is a lack of correlation between corneal and total ocular HOAs ; thereby, this raised the query if we can depend on corneal HOAs obtained from corneal wavefront map only to plan for refractive surgery $(12)(13)(14)(15)$.

The aim of this study was to compare the safety, efficacy, and predictability of corneal WFG and aberration free ablation in TransPRK for myopic patients with or without astigmatism with high preexisting corneal HOAs.

\section{Methods}

This was a non-randomized controlled trail conducted in El-Hekma Eye-LASIK center, Menoufia governorate, Egypt. The study methods adhered to the tenets of the Declaration of Helsinki for use of human participants in biomedical research and were approved by ethical committee of Menoufia medical college. The assignment of patients was done according to the expected ablation depth and estimated residual stromal bed thickness with allocation ratio 2:1 in aberration free and corneal WFG groups respectively.

Enrollment in the study was restricted to those 21 years or older with stable refraction for more than 1 year, off soft contact lens for minimum 14 days prior to preoperative examination, preoperative central corneal thickness at least $480 \mu \mathrm{m}$, estimated residual stromal bed thickness at least $350 \mu \mathrm{m}$ and total corneal HOAs $\geq 0.35 \mu$.

Exclusion included any patient with any of the followingocular diseases; severe dry eye, blepharitis, corneal disease, contact lens warpage, cataract, uveitis and posterior segment anomalies involving the macula or optic nerve or any of the following systemic conditions; diabetes mellitus, connective tissue disease, pregnancy or nursing. Also, any patient who had previous ocular surgery including keratorefractive surgery was excluded.

Preoperative assessment included uncorrected distance visual acuity (UDVA) andbest spectacle corrected distance visual acuity (CDVA) utilizing Landolt $C$ chart expressed in 20/20 and decimal notations for statistical analysis, manifest refraction and cycloplegic refractions using Topcon auto-refractometer 5500 (Topcon Inc., Japan), corneal topography, thorough slit lamp examination, and intraocular pressure measurement using Goldmanapplanation tonometer done after manifest refraction, CDVA and corneal topography to avoid bias. Corneal topography was carried out utilizing corneal wavefront topographer Schwind Sirius (SCHWIND eye-tech-solutions, Kleinhostheim, Germany) which combines a rotating scheimpflug camera with a placido disc and involves integrated pupillometry. Corneal wavefront map of the anterior corneal surface was obtained from corneal wavefront topographerSchwind Sirius up to the 
7th order of Zernike polynomial at $6 \mathrm{~mm}$ diameter and expressed as root mean square (RMS) values, measured in micrometer $(\mu \mathrm{m})$ mainly total, coma, spherical and trefoil aberrations. These measures were repeated 6 months postoperatively.

In this study, a procedure was labeled successful if achieved the desired outcomes in terms of efficacy and safety.Efficacy index was defined as the ratio between the postoperative UDVA to the preoperative CDVA while safety index was defined asthe ratio between the postoperative CDVA and the preoperative CDVA. Efficacy index around 1 and safety index more than 1 were desired. Failure was considered when the above outcomes failed to be achieved along with persistence or development of any of the following complications at 6 month postoperative follow up; corneal haze, persistent epithelial defects, ectasia, sterile infiltrate, infectious keratitis, central toxic keratopathy and steroid induced complications.

\section{Planning for surgery}

Organized Refractive Keratectomy Custom Ablation Manager (ORK CAM) software (SCHWIND eye-techsolutions, Kleinostheim, Germany) was used to plan corneal wavefront customized treatments and aberration free aspheric treatments. Aberration free aspheric treatment involved ablation with optimized aspheric profile centered on the pupil center and the software compensated for the peripheral loss of energy while corneal wavefront mode involved ablation with corneal topography centered on the corneal vertex. Emmetropia was targeted in all patients.

\section{Surgical technique}

All patients underwent TransPRK using excimer laser system (SCHWIND AMARIS ${ }^{\circ} 500 E$, SCHWIND eyetech-solutions, Kleinhostheim, Germany), with $500 \mathrm{~Hz}$ repetition rate for faster treatments and scanning spots and with smart pulse technology. Eye-tracking during laser ablation was achieved using a $1050 \mathrm{~Hz}$ infrared eye tracker centered on the pupil. Static cyclotorsion control (SCC) compensation was obtained for all patients in corneal WFG group and patients with astigmatism more than $1 \mathrm{D}$ in aberration free group. Dynamic cyclotorsion control was implemented automatically for all treatment types. The optical zone was chosen by the surgeon. It ranged from $6 \mathrm{~mm}$ to $7 \mathrm{~mm}$ in aberration free group and $6.5 \mathrm{~mm}$ to 6.8 $\mathrm{mm}$ in corneal WFG group. The transitional zone was determined by ORKCAM software.

After the patient being prepped, topical Benoxinate hydrochloride (BENOX®0.4\%, Sterile Ophthalmic Solution, Eipico, Egypt) anesthetic drops were placed in the eye. Lid speculum was placed in the operative eye and a patch was placed over the fellow eye to avoid cross-fixation. The amount of desired correction, accounting for the vertex distance, was entered into the laser and the patient was asked to fixate on the laser centration light, then the excimer laser was used to remove the epithelium estimating that the central epithelial thickness of a normal cornea is $55 \mu$ at the center and $65 \mu$ at $4 \mathrm{~mm}$ from the center, then continued to ablate the corneal stroma in non-touch technique. Topical Mitomycin-C (Mitomycin-C, kyowa Inc., Japan) $0.2 \mathrm{mg} / \mathrm{ml}$ was employed in all cases according to diopteric correction, with minimum $20 \mathrm{sec}$ 
and maximum $60 \mathrm{sec}$ at the end of the laser exposure followed by generous irrigation of the eye with room temperature balanced salt solution ( 20 seconds was used for corrections less than 3 diopters and 60 seconds for more than 3 diopters). After the surgery, a bandage contact lens was applied.

Postoperative treatment included a broad spectrum antibiotics eye drops,Moxifloxacin $0.5 \%$ (Vigamox, sterile ophthalmic solution, Alcon, USA) and lubricants eye drops,Hyaluronic acid (Hyfresh, JamjoomInc, KSA). Topical steroid drops, Prednisolone acetate 1\% (PredForte, ophthalmic suspension, Allergan, Inc., Irvine, CA, USA), were added on the $2^{\text {nd }}$ day postoperative starting with high frequency for one week, and then tapered gradually over two weeks. The bandage soft contact lens was removed 3-5 days postoperative.

\section{Statistical Analysis}

IBM SPSS for Windows (Version 22.0, Armonk, NY: IBM Corp) was used. Categorical data was expressed as number and percentage, while numerical variables were expressed as mean \pm SD. Normality of data variables were checked. For normally distributed data, preoperative and postoperative values were compared using paired t-test, while student t-test was used to compare the means between both study groups. Corresponding non-parametric tests (Wilcoxon rank test and Mann-Whitney test) were used if data didn't follow a normal distribution. Correlation analysis was conducted between intended and achieved outcomes of interest. For all tests, $p$ value of 0.05 or less was considered statistically significant.

\section{Results}

Thirty-six myopic patients (72 eyes) with or without astigmatism were recruited for this study from January 2017 to December 2017. Three patients (6 eyes) were excluded for having one of the exclusion criteria. Aberration free group included 42 eyes compared to 24 eyes in corneal WFG group.In our study, the mean age was $32.04 \pm 9$ and $25 \pm 3.9$ years for aberration free and corneal WFG group respectively. Female to male ratio was 5:2 and 2:1 in aberration free and corneal WFG group respectively. Demographic characteristics as well as preoperative and operative characteristics showed no statistically significant difference between the two groups except for astigmatism being higher in aberration free group $(p=0.04)$ but not clinically significant and total corneal HOAs being higher in corneal WFG group $(p=0.02)$ [Table 1]. Six months postoperatively, there was no statistically significant difference between the two groups in refractive and visual outcomes [Table 2]. Both groups showed significant improvement in UDVA ( $p=0.001$ and 0.003 in aberration free and corneal WFG groups respectively) with 32 eyes out of 42 eyes $(76.2 \%)$ and 16 eyes out of 24 eyes $(66.67 \%)$ have achieved postoperative UDVA of $\geq 20 / 20$ in aberration free and corneal WFG groups respectively and 36 eyes out of 42 eyes $(85.72 \%)$ and 22 eyes out of 24 eyes (91.67\%) have achieved postoperative CDVA of $\geq 20 / 20$ in aberration free and corneal WFG groups respectively [Figure1]. There was improvement in CDVA postoperatively in both groups ( $p=0.0001$ in both groups). In terms of efficacy, the ratio between postoperative UDVA and preoperative 
CDVA was $1.08 \pm 0.09$ in aberration free group and $1.04 \pm 0.08$ in corneal WFG group with no statistically significant difference between both groups $(p=0.6)$. While mean safety index (the ratio between postoperative CDVA and preoperative CDVA) was $1.16 \pm 0.12$ in aberration free group and $1.17 \pm 0.1$ in corneal WFG group with no statistically significant difference between the two groups $(p=0.9)$ and no eye in either group has showed loss of 1 or more line from the preoperative CDVA. In addition, no postoperative complications were encountered and no patient required retreatment in both groups. Concerning refractive outcome, both groups showed significant change in manifest refraction spherical equivalent (MRSE) ( $p=0.001$ in both groups). $97.62 \%$ (41 eyes out of 42 eyes) and $100 \%$ of patients have achieved postoperative spherical equivalent (SE) within $\pm 1 \mathrm{D}$ in aberration free group and corneal WFG group respectively [Figure 2]. Concerning corneal HOAs, there was increase in the postoperative value of total HOAs and spherical aberrations in both groups that was statistically significant in aberration free group in contrast to corneal WFG, while there was decrease in coma and trefoil aberrations in corneal WFG group in contrast to aberration free group that showed increase in both [Table 3 and Figure 3]. Also, there was significant change in $Q$ value in both groups $(p=0.001$ in both groups) toward more positive values [Table 2] which was positively correlated with the induction of spherical aberrations $(R=0.98$ $p=0.001)$. On the other hand, the induction of spherical aberrations was positively correlated with amount of refractive correction $(R=0.86, p=0.001)$, and negatively correlated with optical zone diameter $(R=-0.56$, $\mathrm{p}=0.001)$. The postoperative central corneal thickness was significantly correlated with expected value preoperatively in both groups $(R=0.92, p=0.001$ in aberration free group, and $R=0.94, p=0.03$ in corneal WFG group). The change in central corneal thickness per 1D treatment applied was $(17.6 \pm 4.6 \mu / D)$ in aberration free group while in corneal WFG group, it was $(20.9 \pm 5.4 \mu / D)$ but not statistically significantly different $(p=0.28)$.

\section{Discussion}

Visual and refractive outcomes of aberration free and corneal WFG treatments were compared after laser in situ keratomileusis $\left(\right.$ LASIK) ${ }^{(16)(17)(18)}$. Moreover, many other studies have compared WFG PRK and wavefrontoptimized PRK based on ocular wave front analysis, not corneal wave front analysis. Different laser platforms were compared, adding more heterogeneity for the outcomes ${ }^{(19)}$. All of this made the comparison difficult and till now, no method has shown superiority over the other. In our study, TransPRK was performed for all patients according to the previously described techniques. With both techniques, satisfactory visual, refractive and optical outcomes were obtained six month postoperatively. In the study by Kaluzny and his colleagues, they reported that TransPRK has achieved good visual results (Postoperative UDVA was $20 / 20$ or better in $97 \%$ compared to $94 \%$ in the alcohol-assisted PRK), as well as good refractive outcomes with high correlation between attempted and achieved manifest refraction spherical equivalent (MRSE) in both groups ${ }^{(20)}$. In another retrospective case series, TransPRK was reported as effective and safe as femtosecond-assisted LASIK at one year postoperatively ${ }^{(1)}$. In our study, $76.2 \%$ and $66.67 \%$ have achieved uncorrected distance visual acuity of $\geq 20 / 20$ in aberration free group and corneal WFG group respectively, six month postoperatively, while in Jun et al. study, $94 \%$ and $99 \%$ have achieved uncorrected distance visual acuity of $\geq 20 / 20$ in aberration free group and corneal WFG 
group respectively ${ }^{(21)}$. Both techniques have also achieved good refractive outcome, with $97.62 \%$ of patients and $100 \%$ of patients have achieved postoperative spherical equivalent (SE) within $\pm 1 \mathrm{D}$ in aberration free group and corneal WFG group respectively. These results are comparable to the result of Jun et al. study in which all treated eyes were within $\pm 1 \mathrm{D}$ of the intended $S \mathrm{E}^{(21)}$. Aslanides and his colleagues reported 2 years refractive and visual outcomes using aberration free TransPRK, with $91 \%$ of eyes were within $0.50 \mathrm{D}$ of the intended MRSE two years postoperatively with postoperative UDVA 20/20 or better in $70 \%$ of patients ${ }^{(22)}$. Concerning optical outcomes, all the patients in our study had preoperative total corneal HOA more than $0.35 \mu \mathrm{m}$ and both groups showed change in total corneal HOAs and spherical aberrations six months postoperatively, with no significant difference in corneal WFG. In contrast, corneal HOAs and spherical aberrations were significantly higher postoperatively in the aberration-free group. Change in corneal HOAs and spherical aberrations value showed no significant difference between both groups. Coma and trefoil aberrations declined after corneal WFG technique, yet increased after aberration free one. Jun et al reported similar findings that confirmed the advantage of corneal WFG over aberrationfree technique regarding the optical outcome, irrespective of the preoperative corneal HOAs as corneal WFG didn't induce trefoil or coma aberrations in contrast to aberrationfree and the increase in spherical and total HOAs were less in corneal WFG ${ }^{(21)}$. Also Aslanides and his colleagues showed that 2 years after aberration free TransPRK, the corneal trefoil and corneal total HOAs increased, but didn't significantly change postoperatively in contrast to increase in postoperative coma and spherical aberrations $^{(22)}$. Other studies have highlighted the role of corneal WFG PRK in cases of highly aberrated cornea as after previous refractive surgery and in keratoconus eyes after cross linking in terms of visual, optical, and refractive outcomes with reduction in the preexisting corneal HOAs$s^{(11)(23)(24)(25)}$. Generally speaking, comparing WFG PRK against wavefront optimized PRK, using different laser platforms, have been debatable, but all agreed that both techniques are safe and effective with subtle differences between the two techniques. In Moshirfar et al study utilizing VISX CustomVue and WaveLight Allegretto platforms, they reported the same efficacy, safety and predictability for the two methods, with comparable significant increase of spherical aberrations with increase in the other HOAs as well. However, better contrast sensitivity was reported in the WFG group ${ }^{(26)}$. Also, in comparing visual outcomes following Visx Star S4 CustomVue WFG and Allegretto Wave Eye-Q $400 \mathrm{~Hz}$ wavefront optimized PRK, both techniques were similarly effective and safe, with better contrast visual acuity obtained with the WFG technique ${ }^{(27)}$. Another study detected an increase in HOA values postoperatively after both technique with significantly lower coma and trefoil aberrations and astigmatism in WFG group ${ }^{(28)}$. Contrast sensitivity was always a concern after any refractive procedures. In military settings, contrast sensitivity is a central point to consider. Both ablation procedures were tested in military personnel who were evaluated for contrast sensitivity and target hitting after the procedure. Fortunately, satisfactory visual performance and contrast sensitivity was achieved after both techniques ${ }^{(29)(30)}$. Nonetheless, other studies showed superiority of aberration free over WFG ablation in PRK. In ZareiGhanavati and his colleagues study, they reported improved contrast sensitivity after both procedures with significantly lower postoperative HOAs in aberration free group, particularly the spherical aberrations $^{(19)}$. In our study, there was significant positive correlation between induction of spherical 
aberrations and the change in spherical equivalent, and significant negative correlation with the change in optical zone diameter. However, no significant correlation was detected with the coma aberrations. This correlation was proved by other studies after LASIK and LASEK (Laser epithelial keratomileusis) ${ }^{(31)(32)}$. This indicates that larger optical and transitional zones are recommended to minimize the induction of spherical aberrations or combining the surgical technique with $Q$ value customized treatments especially with higher refractive errors. To our knowledge, this was the first comparison between corneal WFG and aberration free ablation techniques in TransPRK in myopic patients with high pre-existing corneal HOAs. However, limitations exist in the study. Absence of data about contrast sensitivity testing and other subjective assessment of visual quality detract from the comparison. Moreover, lack of cross matching between the two groups may allow confounders to have a place.One major criticism is the fact that the two groups did not have similar cylinder preoperatively and they did not have similar corneal HOA preoperatively. So, a larger well-designed study with matched groups that combines objective and subjective outcomes is strongly recommended.

\section{Conclusion}

Both aberration free and corneal WFG ablation profiles in TransPRK were safe, effective, and predictable techniques in treatment of myopia with or without astigmatism in patients with high pre-existing corneal HOA measured at $6 \mathrm{~mm}$ diameter. Comparable visual and refractive outcomes were obtained. However, Corneal WFG treatment has the advantage of induction of fewer total corneal HOAs and spherical aberrations with reduction of postoperative coma and trefoil aberrations in contrast to aberration free treatment.

\section{Abbreviations}

WFG: Wavefront guided

PRK: Photorefractive keratectomy

TransPRK : Transepithelial photorefractive keratectomy

HOAs:Higher order aberrations

\section{Declarations}

Ethics approval and consent to participate: The study methods adhered to the tenets of the Declaration of Helsinki for use of human participants in biomedical research and were approved by ethical committee of Menoufia medical college.

Consent for publication: Not applicable

Availability of data and material: are available on need by corresponding author 
Authors' contributions:

AF performed the surgical procedures and postoperative data

$\mathrm{MZ}$ analyzed and interpreted the patient data and analysis of all parameters.

RA was a major contributor in writing the manuscript.

All authors read and approved the final manuscript

Competing interests: No

Funding: No

Acknowledgements: Not applicable

ClinicalTrials.gov ID:NCT03786861

\section{References}

1. Luger MHA, Ewering T, Arba-Mosquera S. Myopia correction with transepithelial photorefractive keratectomy versus femtosecond-assisted laser in situ keratomileusis: One-year case-matched analysis. J Cataract Refract Surg. 2016;42(11):1579-87.

2. Fadlallah A, Fahed D, Khalil K, Dunia I, Menassa J, El Rami H, et al. Transepithelial photorefractive keratectomy: Clinical results. J Cataract Refract Surg. 2011;37(10):1852-7.

3. Oshika T, Klyce SD, Applegate RA, Howland HC, El Danasoury MA. Comparison of corneal wavefront aberrations after photorefractive keratectomy and laser in situ keratomileusis. Am J Ophthalmol. 1999;127(1):1-7.

4. Wang Y, Zhao KX, He JC, Jin Y, Zuo T. Ocular higher-order aberrations features analysis after corneal refractive surgery. Chin Med J (Engl). 2007;120(4):269-73.

5. Seiler T, Kaemmerer M, Mierdel P, Krinke HE. Ocular optical aberrations after photorefractive keratectomy for myopia and myopic astigmatism. Arch Ophthalmol. 2000;118(1):17-21.

6. Tanabe T, Miyata K, Samejima T, Hirohara Y, Mihashi T, Oshika T. Influence of wavefront aberration and corneal subepithelial haze on low-contrast visual acuity after photorefractive keratectomy. Am J Ophthalmol. 2004;138(4):620-4.

7. Mrochen M, Seiler T. Influence of corneal curvature on calculation of ablation patterns used in photorefractive laser surgery. J Refract Surg. 2001;17(5):S584-7. 
8. Koller T, Iseli HP, Hafezi F, Mrochen M, Seiler T. Q-factor customized ablation profile for the correction of myopic astigmatism. J Cataract Refract Surg. 2006;32(4):584-9.

9. Kohnen T. Classification of excimer laser profiles. Journal of Cataract and Refractive Surgery. 2006;32(4):543-4.

10. Pasquali T, Krueger R. Topography-guided laser refractive surgery. Vol. 23, Current Opinion in Ophthalmology. 2012. p. 264-8.

11. Alió JL, Piñero DP, Plaza Puche AB. Corneal wavefront-guided photorefractive keratectomy in patients with irregular corneas after corneal refractive surgery. J Cataract Refract Surg. 2008;34(10):1727-35.

12. Mehta CK. Corneal-Wavefront-Guided LASIK for Highly Irregular Corneas. Cataract \&Refractive Surgery Today Europe. 2008. p. 59-62.

13. Mrochen M, Jankov M, Bueeler M, Seiler T. Correlation between corneal and total wavefront aberrations in myopic eyes. J Refract Surg. 2003;19(2):104-12.

14. Amano S, Amano Y, Yamagami S, Miyai T, Miyata K, Samejima T, et al. Age-related changes in corneal and ocular higher-order wavefront aberrations. Am J Ophthalmol. 2004;137(6):988-92.

15. Athaide HVZ, Campos M, Costa C. Study of ocular aberrations with age. Arq Bras Oftalmol. 2009;72(5):617-21.

16. Padmanabhan P, Mrochen M, Basuthkar S, Opto M, Viswanathan D, Joseph R. Wavefront-guided versus wavefront-optimized laser in situ keratomileusis: Contralateral comparative study. 2007;389-97.

17. Miraftab M, Seyedian MA, Hashemi H. Wavefront-Guided vs Wavefront- Optimized LASIK: A Randomized Clinical Trial Comparing Contralateral Eyes. 2011;33.

18. He L, Liu A, Manche EE. Wavefront-Guided Versus Wavefront-Optimized Laser In Situ Keratomileusis for Patients with Myopia: A Prospective Randomized Contralateral Eye Study. Am J Ophthalmol. 2014.

19. Zarei-Ghanavati S, Gharaee H, Hamilton DR, Sanchez PJ, Abrishami M. Aspheric versus wavefrontguided photorefractive keratectomy: Contralateral eye study. J Cataract Refract Surg. 2015;41(7):1441-7.

20. Kaluzny BJ, Cieslinska I, Mosquera SA, Verma S. Single-Step Transepithelial PRK vs Alcohol-Assisted PRK in Myopia and Compound Myopic Astigmatism Correction. Med (United States). 2016;95(6).

21. Jun I, Kang DSY, Tan J, Choi JY, Heo W, Kim JY, et al. Comparison of clinical outcomes between wavefront-optimized versus corneal wavefront-guided transepithelial photorefractive keratectomy for myopic astigmatism. J Cataract Refract Surg. 2017;43(2):174-82.

22. Aslanides IM, Padroni S, Arba-Mosquera S. Aspheric photorefractive keratectomy for myopia and myopic astigmatism with the SCHWIND AMARIS laser: 2 years postoperative outcomes. J Optom. 
2013;6(1):9-17.

23. Ghanem RC, Ghanem VC, Ghanem EA, Kara-José N. Corneal wavefront-guided photorefractive keratectomy with mitomycin-C for hyperopia after radial keratotomy: Two-year follow-up. J Cataract Refract Surg. 2012;38(4):595-606.

24. Camellin M, Guidotti JM, Arba Mosquera S. Corneal-Wavefront guided transepithelial photorefractive keratectomy after corneal collagen cross linking in keratoconus. J Optom. 2017;10(1):52-62.

25. Munger R. Corneal wavefront-guided retreatments for significant night vision symptoms after myopic laser refractive surgery. Vol. 9, Evidence-Based Ophthalmology. 2008. p. 228-9.

26. Moshirfar M, Churgin DS, Betts BS, Hsu M, Sikder S, Neuffer M, et al. Prospective, randomized, fellow eye comparison of WaveLight ${ }^{\circledR}$ Allegretto Wave ${ }^{\circledR}$ Eye-Q versus VISX CustomVue ${ }^{\text {TM }}$ STAR S4 IR ${ }^{\text {TM }}$ in photorefractive keratectomy: Analysis of visual outcomes and higher-order aberrations. Clin Ophthalmol. 2011;5(1):1185-93.

27. Sia RK, Ryan DS, Stutzman RD, Pasternak JF, Eaddy JB, Logan LA, et al. Wavefront-guided versus wavefront-optimized photorefractive keratectomy: Clinical outcomes and patient satisfaction. J Cataract Refract Surg. 2015;41(10):2152-64.

28. He L, Manche EE. Contralateral eye-to-eye comparison of wavefront-guided and wavefront-optimized photorefractive keratectomy: A randomized clinical trial. JAMA Ophthalmol. 2015;133(1):51-9.

29. Maurer T, Deaver D, Howell C, Moyer S, Nguyen O, Mueller G, et al. Military target task performance after wavefront-guided (WFG) and wavefront-optimized (WFO) photorefractive keratectomy (PRK). Proc SPIE - Int Soc Opt Eng. 2014;9112:91120U.

30. Ryan DS, Sia RK, Stutzman RD, Pasternak JF, Howard RS, Howell CL, et al. Wavefront-Guided Versus Wavefront-Optimized Photorefractive Keratectomy: Visual and Military Task Performance. Mil Med. 2017;182(1):e1636-44.

31. Piao JJ, Li YJ, Whang WJ, Choi M, Kang MJ, Lee JH, et al. Comparative evaluation of visual outcomes and corneal asphericity after laser-assisted in situ keratomileusis with the six-dimension Amaris excimer laser system. PLoS One. 2017;12(2):1-11.

32. Huang $\mathrm{H}$, Yang J, Bao H, Chen S, Xia B, Zou J. Retrospective analysis of changes in the anterior corneal surface after $\mathrm{Q}$ value guided LASIK and LASEK in high myopic astigmatism for 3years. BMC Ophthalmol 2012;12(1):1.

\section{Tables}


Due to technical limitations, Table(s) 1-3 are only available as a download in the supplemental files section.

\section{Figures}

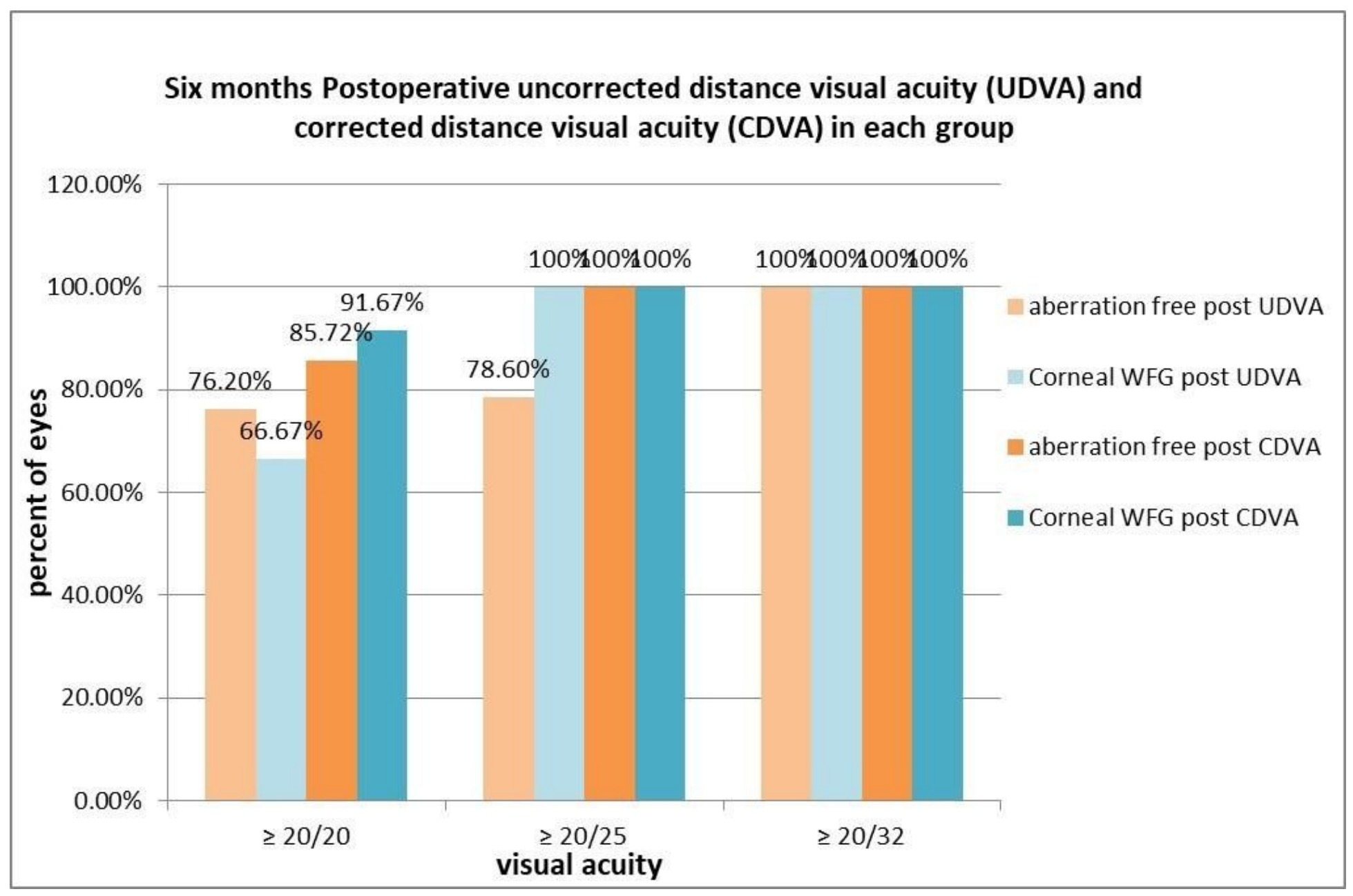

\section{Figure 1}

Six months postoperative uncorrected distance visual acuity (UDVA) and corrected distance visual acuity (CDVA) in each group 
Six months postoperative manifest refraction spherical equivalent

(MRSE) in each group

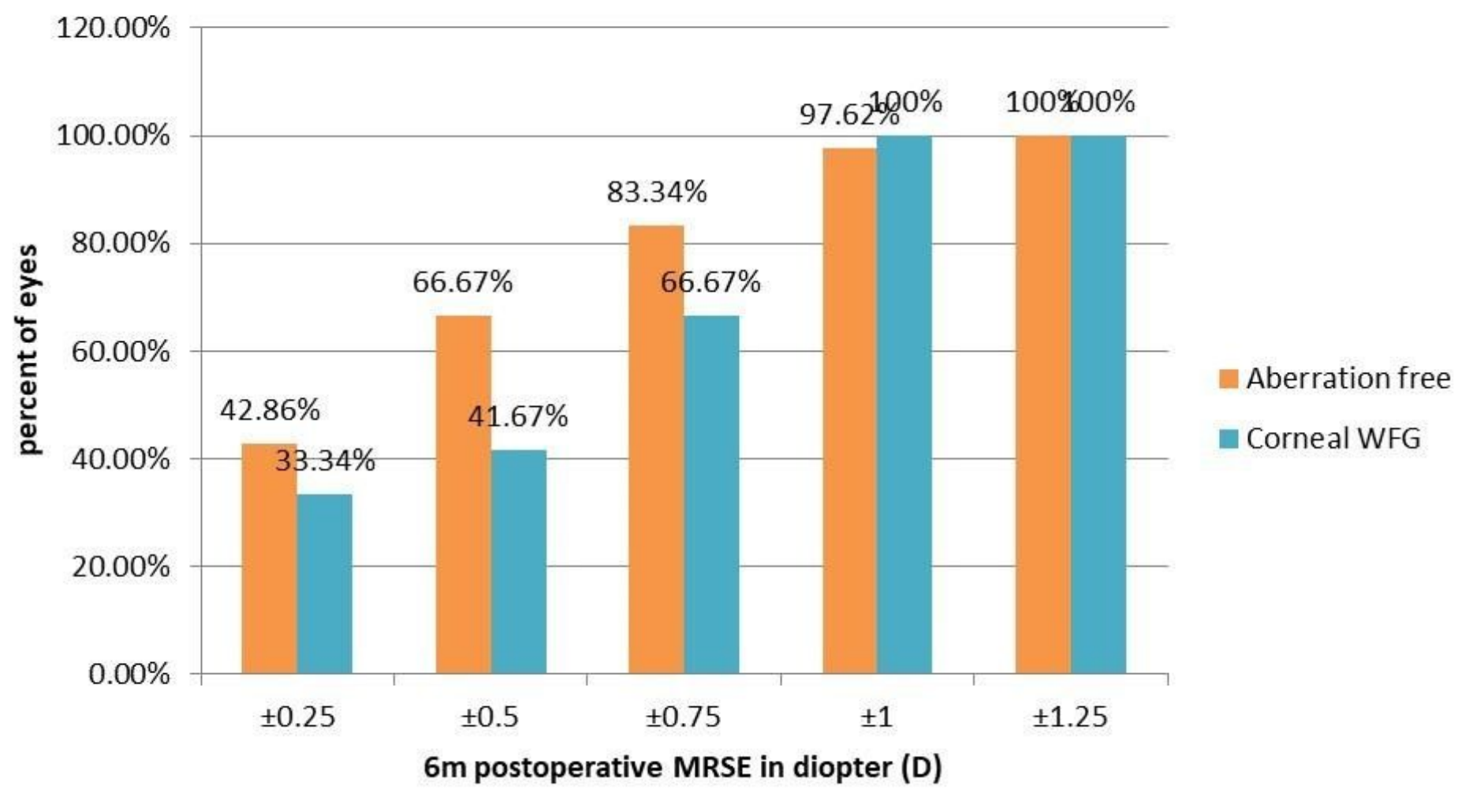

Figure 2

Six months postoperative manifest refraction spherical equivalent (MRSE) in each group

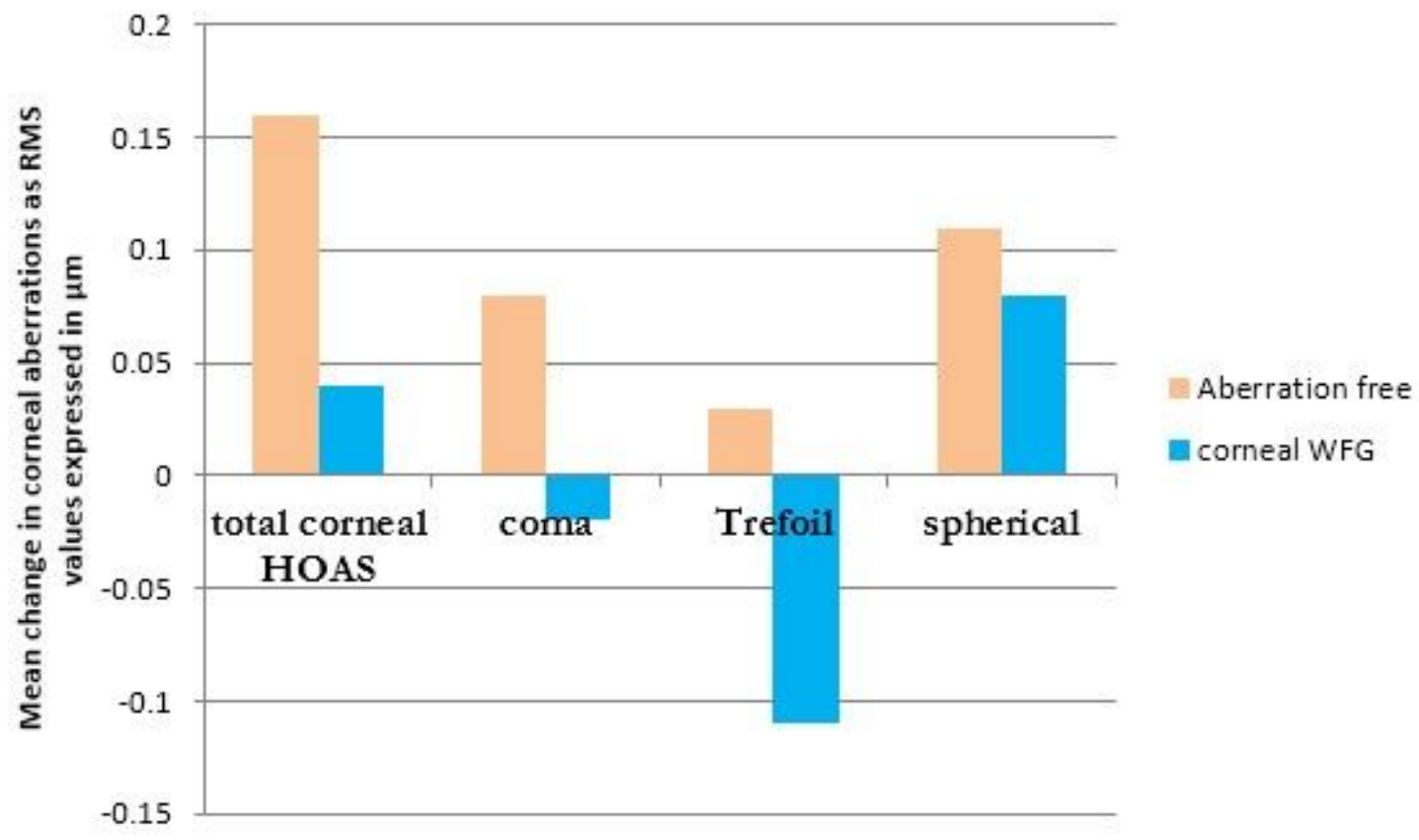

Figure 3 
Mean change in corneal higher order aberrations (HOAs) in each group

\section{Supplementary Files}

This is a list of supplementary files associated with this preprint. Click to download.

- supplement1.doc

- supplement2.pdf

- supplement3.pdf

- supplement4.pdf 